\title{
UNE EXPÉRIENCE D'ESPACEMENT DE PLANTATION DE SAPIN DE VANCOUVER (Abies grandis)
}

\author{
H. OSWALD
}

Le sapin de Vancouver (Abies grandis (Dougl.) Lindl.) ou sapin géant est originaire des régions occidentales de l'Amérique du Nord. Schématiquement, on peut distinguer une espéce côtière qui s'étend du sud-ouest de la Colombie Britannique (lle de Vancouver) jusqu'au nord de la Californie et, séparée par des hauts plateaux très secs, une espèce intérieure du sud-est de la Colombie Britannique, le nord de l'Idaho et le nord-ouest du Montana (Hermann, 1978).

Introduite en Europe à la fin du siècle dernier, surtout dans les "arboreta", on ne trouve aujourd'hui en France que très peu de peuplements purs et âgés, d'une certaine superficie. Entre 1945 et 1970, plusieurs milliers d'hectares ont été reboisés avec cette essence en France et on commença à mettre en place des dispositifs expérimentaux pour étudier la variabilité génétique des différentes provenances (Lacaze et Tomassone, 1967), la production (Pardé, 1960 - Prax et Pardé, 1964), et la sylviculture.

Dans le cadre de ces études, une expérience d'espacement à la plantation fut mise en place en 1958 en forêt domaniale d'Amance, près de Nancy, dans le nord-est de la France. Nous possédons maintenant, 20 ans après la plantation, un certain nombre de résultats qui peuvent intéresser les forestiers.

\section{DESCRIPTION DU DISPOSITIF}

Le dispositif est installé dans les parcelles 49 et 50 de la forêt domaniale d'Amance, à environ $250 \mathrm{~m}$ d'altitude. La pluviosité annuelle est de $687 \mathrm{~mm}$, bien répartie sur toute l'année. La température moyenne annuelle est de $9,2^{\circ} \mathrm{C}$; des gelées tardives sont à craindre.

Le type de sol est un sol brun lessivé, à pseudogley, développé sur des marnes liasiques recouvertes de limons. Une couche très compacte vers $65 \mathrm{~cm}$ de profondeur provoque l'apparition d'une nappe perchée temporaire. L'horizon $A_{1}$ est un mull mésotrophe à pH 5,5. Le sol est riche en éléments échangeables et en phosphore; la minéralisation de l'azote est rapide. 
Le peuplement d'origine était un taillis sous futaie trés endommagé par les deux guerres et dont on avait extrait en 1948 toutes les réserves (chênes et hêtres) d'un diamètre supérieur à $20 \mathrm{~cm}$.

Dans ce peuplement restant âgé d'environ 30 ans, une coupe d'abri fut réalisée dans l'hiver 1957 1958, laissant sur pied à l'hectare un couvert de :

- 748 brins (circonférence moyenne : $23 \mathrm{~cm}$ ) composés de :

- $58 \%$ de charmes,

- $34 \%$ de tilleuls,

- $8 \%$ de hêtres, trênes et chênes.

- 70 modernes (circonférence moyenne : $60 \mathrm{~cm}$ ) composés de :

- $60 \%$ de charmes,

- $20 \%$ de hêtres,

- $20 \%$ de tilleuls et autres.

II faut ici souligner le fait qu'á cette époque les enrésinements en sapins se faisaient encore exclusivement sous couvert.

La plantation, en potets creusés au préalable, a été faite au printemps 1958, avec des plants de 3 ans $(1+2)$. Les plants ont été élevés dans la pépinière de l'arboretum de Pezanin (Saône-etLoire), d'où proviennent également les graines. II s'agit d'une provenance artificielle française, probablement originaire de la région côtière, comme l'ont montré les expériences comparatives de provenances (Lacaze et Tomassone, 1967).

Le dispositif est un carré latin $4 \times 4$ classique, comportant 16 placettes unitaires d'environ 10 ares, chacune étant entourée d'une zone de protection de 4 à $7 \mathrm{~m}$. L'ensemble du dispositit qui s'étend sur 3,3 ha fut clôturé.

Les traitements suivants ont été retenus :

- 4 carrés B plantés à $2,00 \mathrm{~m} \times 2,00 \mathrm{~m}$ (2 500 plants/ha)

- 4 carrés $\mathrm{C}$ plantés à $2,50 \mathrm{~m} \times 2,50 \mathrm{~m}$ (1 600 plants/ha)

- 4 carrés D plantés à $3,00 \mathrm{~m} \times 3,00 \mathrm{~m}$ (1111 plants/ha)

- 4 carrés $E$ plantés à $3,50 \mathrm{~m} \times 3,50 \mathrm{~m}$ (816 plants/ha)

Des regarnis portant sur environ $20 \%$ des plants ont été effectués au printemps 1959 et 1960 .

Le couvert teuillu fut progressivement enlevé entre 1961 et 1965 :

- en février 1961: $15 \%$ des brins exploités (souches traitées au P 80 ), plus $25 \%$ des brins traités au $\mathrm{P} 80$;

- en mai 1962 : exploitation de $5 \%$ des brins, plus les brins traités en 1961 ;

- en mars 1965: enlévement total du couvert restant (43\%).

Les dégagements suivants ont été exécutés :

- printemps 1959 : dégagements (herbes, ronces) autour des potets;

- hiver 1961/62: piochage des potets (herbes et ronces);

- automne 1962 : dégagements (rejets gênants);

- été 1963 : dégagements (rejets gênants) ;

- printemps 1966 : dégagements (tous les rejets);

- hiver 1971/72: dégagements (tous les rejets).

II faut donc retenir que la plantation est restée sous le couvert pendant 7 périodes de végétation et qu'au moins 6 dégagements pendant 10 à 14 ans sont nécessaires, avant que la fermeture du couvert dans les larges espacements devienne suffisante pour empêcher complètement le développement des rejets et de la ronce. 


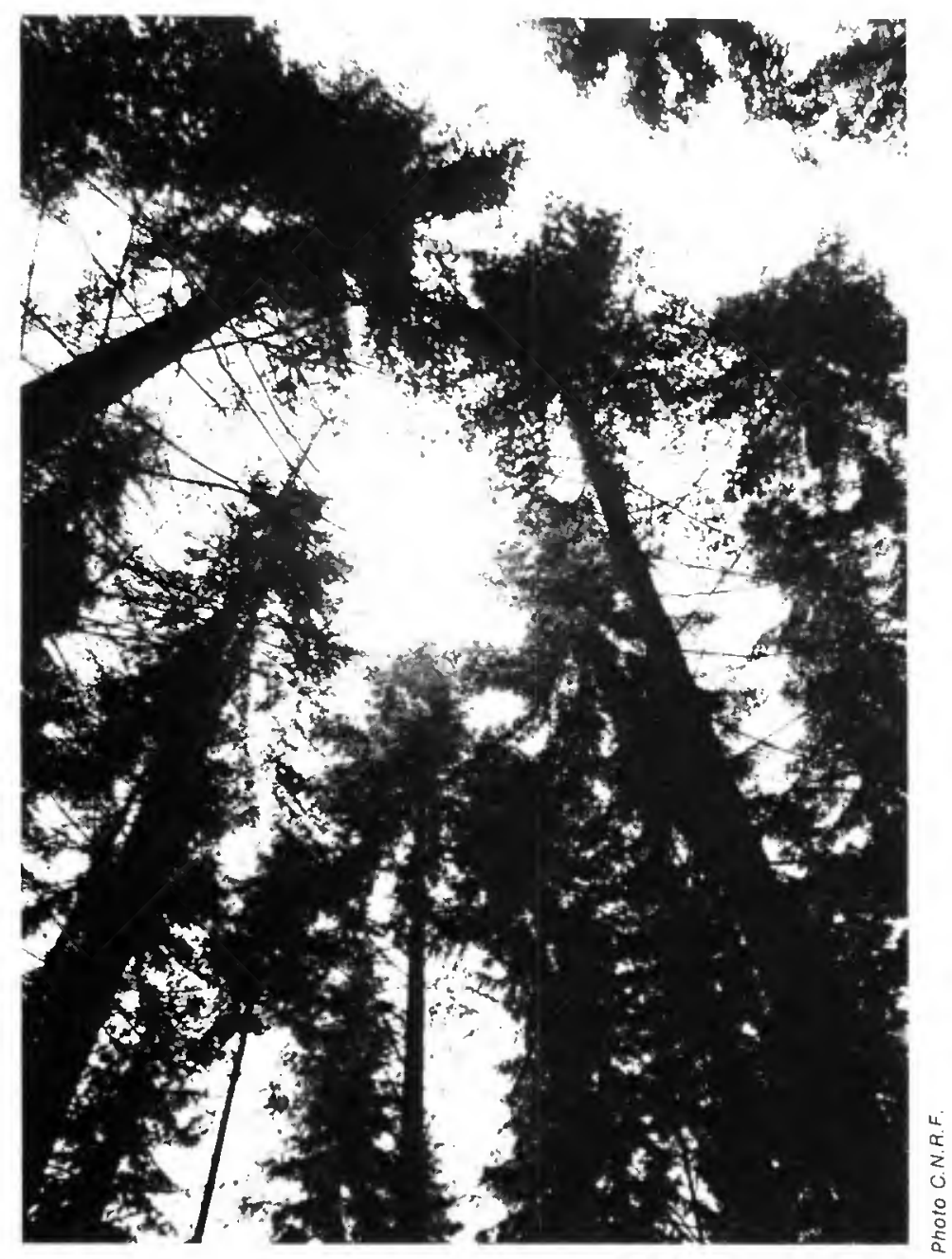

\section{CROISSANCE ET PRODUCTION}

Des mesures dendrométriques ont été effectuées en 1966, 1974 et 1977. L'ensemble de ces données a èté traité dans le cadre du self-service à la station de Biométrie et de Calcul automatique de I'I.N.R.A.-C.N.R.F." en utilisant la chaîne de programmes de l'analyse de variance (Badia et Masson, 1977).

Les principaux résultats des inventaires 1966, 1974 et 1977 sont condensés dans le tableau 1.

- Institut national de la Recherche Agronomique - Centre national de Recherches forestieres. 


\begin{tabular}{|c|c|c|c|c|c|}
\hline \multirow{3}{*}{ Variables étudiées } & \multicolumn{4}{|c|}{ TRAITEMENTS } & \multirow{3}{*}{$\begin{array}{c}\text { Ditférences } \\
\text { significatives } \\
\text { (Seuil } \leqslant 5 \%) \\
\text { NS : non significatif } \\
\text { * : } \text { significatif à } 5 \% \\
\text { * : significatif à } 1 \%\end{array}$} \\
\hline & B & C & D & $\mathbf{E}$ & \\
\hline & $\begin{array}{l}2,0 \times 2,0 \mathrm{~m} \\
2500\end{array}$ & $\begin{array}{c}2,5 \times 2,5 \mathrm{~m} \\
1600\end{array}$ & $\begin{array}{c}3,0 \times 3,0 \mathrm{~m} \\
1111\end{array}$ & $\begin{array}{l}3,5 \times 3,5 \mathrm{~m} \\
816\end{array}$ & \\
\hline \multicolumn{6}{|c|}{$\begin{array}{l}\text { INVENTAIRE 1966, } 9 \text { annèes après la plantation } \\
\text { (âge : } 12 \text { ans) }\end{array}$} \\
\hline 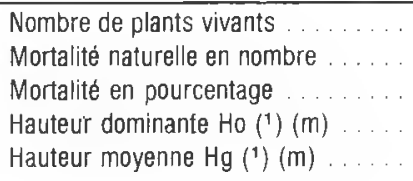 & $\begin{array}{c}2317 \\
184 \\
7,3 \\
4,08 \\
2,31\end{array}$ & $\begin{array}{l}1494 \\
106 \\
6,6 \\
3,95 \\
2,29\end{array}$ & $\begin{array}{c}1027 \\
84 \\
7,6 \\
3,97 \\
2,39\end{array}$ & $\begin{array}{l}745 \\
71 \\
8,7 \\
3,55 \\
2,30\end{array}$ & $\begin{array}{l}- \\
- \\
\text { NS } \\
\text { NS }\end{array}$ \\
\hline \multicolumn{6}{|c|}{$\begin{array}{l}\text { INVENTAIRE 1974, } 17 \text { années après la plantation } \\
\text { (âge : } 20 \text { ans) }\end{array}$} \\
\hline 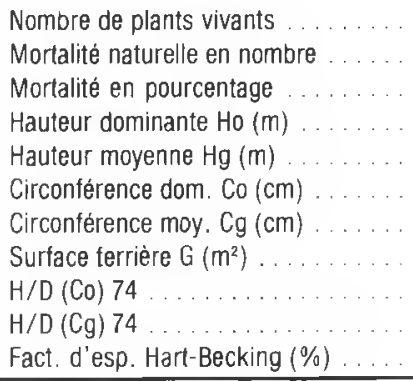 & $\begin{array}{l}2071 \\
246 \\
10,6 \\
12,09 \\
9,49 \\
66,9 \\
39,4 \\
25,77 \\
56,7 \\
75,4 \\
19,5\end{array}$ & $\begin{array}{c}1445 \\
49 \\
3,3 \\
12,01 \\
9,61 \\
70,4 \\
43,4 \\
21,74 \\
53,8 \\
69,6 \\
23,5\end{array}$ & $\begin{array}{l}964 \\
63 \\
6,1 \\
12,33 \\
9,75 \\
75,9 \\
47,6 \\
17,57 \\
51,2 \\
64,3 \\
28,1\end{array}$ & $\begin{array}{c}727 \\
18 \\
2,4 \\
11,44 \\
9,36 \\
72,3 \\
48,2 \\
13,92 \\
49,7 \\
61,3 \\
34,8\end{array}$ & $\begin{array}{l}- \\
- \\
N S \\
N S \\
N S \\
N S \\
* * E D \neq B, E \neq C \\
* * E \neq B C, D \neq B \\
* E D \neq B, C \neq E \\
* E \neq B \neq C \neq D \\
-E\end{array}$ \\
\hline \multicolumn{6}{|c|}{$\begin{array}{l}\text { INVENTAIRE 1977, } 20 \text { années après la plantation } \\
\text { (âge : } 23 \text { ans) }\end{array}$} \\
\hline Nombre de plants vivants $\ldots \ldots \ldots$ & 1991 & 1432 & 957 & 717 & - \\
\hline Mortalité naturelle en nombre. & 80 & 13 & 7 & 10 & - \\
\hline Mortalité en pourcentage .... & 4,0 & 0,9 & 0,7 & 1,4 & NS \\
\hline Mortalité depuis l'origine (\%). & 20.4 & 10,5 & 13,9 & 12,1 & NS \\
\hline Hauteur dominante $\mathrm{Ho}(\mathrm{m}) \ldots$ & 14,73 & 14,57 & 14,66 & 13,96 & NS \\
\hline Hauteur moyenne $\mathrm{Hg}(\mathrm{m}) \ldots$ & 12,23 & 11,96 & 12,20 & 12,10 & NS \\
\hline Circonférence dom. Co $(\mathrm{cm})$.. & 79,8 & 84,8 & 92,8 & 89,9 & NS \\
\hline Circonférence moy. $\mathrm{Cg}(\mathrm{cm}) \ldots$ & 46,6 & 53,0 & 59,2 & 62,1 & $* * E D \neq C \neq B$ \\
\hline Surtace terrière $\mathrm{G}\left(\mathrm{m}^{2}\right) \ldots \ldots$ & 35,33 & 32,0 & 26,72 & 22,53 & $\star E D \neq B C$ \\
\hline Volume bois tige VTT $\left(\mathrm{m}^{3}\right) \ldots$ & 176,10 & 139,10 & 115,15 & 88,58 & $* E^{*} \neq B \neq C, D \neq B$ \\
\hline Volume arbre moyen VM $\left(\mathrm{dm}^{3}\right) \ldots$ & 90,10 & 97,77 & 121,40 & 120,64 & $* \mathrm{ED} \neq \mathrm{BC}$ \\
\hline Coefficient de forme $\left({ }^{2}\right) F \ldots \ldots \ldots$ & 0,3976 & 0,3624 & 0,3468 & 0,3165 & ${ }^{\star} E \neq B \neq C, D \neq B$ \\
\hline Taux d'accr. en surf. terr. (\%) & 1,3914 & 1,4771 & 1,5399 & 1,6473 & $* * E \neq C D \neq B$ \\
\hline Accr. surf. terr. 74 à $77\left(\mathrm{~m}^{2}\right) \ldots$ & 9,57 & 10,27 & 9,15 & 8,61 & \\
\hline Accr. moy, VTT depuis l'orig. $\left(\mathrm{m}^{3}\right) \ldots$ & 7,66 & 6,05 & 5,01 & 3,85 & $* \star E \neq B \neq C, D \neq B$ \\
\hline $\mathrm{H} / \mathrm{O}(\mathrm{Co}) 77 \ldots \ldots \ldots \ldots \ldots \ldots$ & 57,9 & 54.1 & 49,7 & 48,8 & $* \star E D \neq C \neq B$ \\
\hline $\mathrm{H} / \mathrm{D}(\mathrm{Cg}) 77 \ldots \ldots \ldots \ldots \ldots$ & 82,1 & 70,9 & 64,8 & 61,4 & $* * \mathrm{ED} \neq \mathrm{C} \neq \mathrm{B}$ \\
\hline Fact. d'esp. Hart-Becking (\%) . . . . & 16,3 & 19,5 & 23,7 & 28,7 & - \\
\hline
\end{tabular}

(1) Moyenne arithmétique.

(2) Coefficient de forme au peuplement, relatif au volume commercial bois tige total (VTT) $\mathrm{Hg}$ et $\mathrm{G}$. F $=\mathrm{VTT} /(\mathrm{Hg} \times \mathrm{G})$ 


\section{La mortalité naturelle}

En 1966, après l'enlèvement total de l'abri, la mortalité est sensiblement la même (7 à $9 \%$ ) dans les quatre traitements.

Jusqu'à 1977 , elle augmente un peu plus dans le traitement $B$ le plus dense $(20,4 \%)$, inais á cause de la grande variabilité, l'effet n'est pas encore significatif. La mortalité n'est encore que partiellement due à la concurrence.

\section{Hauteurs moyennes et dominantes}

En 1966, la hauteur totale de tous les arbres a été mesurée et les hauteurs moyennes et dominantes sont des moyennes arithmétiques de la population correspondante (les 100 plus hauts arbres à l'hectare, pour la hauteur dominante).

La figure 1 représente la répartition des tiges, par classes de hauteur de $50 \mathrm{~cm}$ d'intervalle et on

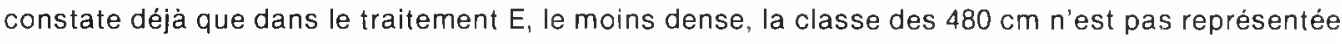
et l'arbre le plus haut n'a que $425 \mathrm{~cm}$. La différence entre la hauteur moyenne et la hauteur dominante en 1966 est d'ailleurs significativement plus petite de $52 \mathrm{~cm}$ par rapport au traitement $\mathrm{B}$.

Le coefficient de variation des hauteurs en 1966 est d'environ $33 \%$, quel que soit le traitement.

Les hauteurs en 1974 et en 1977 ont été déterminées d'après une courbe hauteur/circonférence, établie pour chaque placette unitaire, à partir d'un échantillon représentatif d'une trentaine d'arbres.

Les hauteurs dominantes 1974 et 1977 ne sont pas significativement différentes dans les quatre traitements. En 1977, la hauteur dominante du traitement $E$ est cependant de $77 \mathrm{~cm}(5,2 \%)$ inférieure à celle du traitement $\mathrm{B}$.

Dès 1966, on constate un certain retard de la hauteur dominante du traitement $E$, l'espacement le plus large, par rapport au traitement $B$.

Une analyse de covariance, utilisant comme co-variable la hauteur moyenne en 1966, a été faite pour certaines variables, afin d'éliminer une éventuelle influence d'une inégalité du couvert. Elle apporte une meilleure discrimination, uniquement pour la hauteur dominante 1966 oủ la différence entre $E$ et $B(52 \mathrm{~cm})$ devient alors significative.

Les hauteurs moyennes ne varient que très peu d'un traitement à l'autre, aussi bien en 1966 qu'en 1977. La figure 3 représente les courbes moyennes des hauteurs des traitements E et B. Malgré leurs allures assez différentes, elles n'indiquent qu'une tendance à cause de la grande dispersion des valeurs individuelles.

\section{Circonférences moyennes et dominantes}

La circonférence moyenne est liée à l'espacement, et cet effet augmente de 1974 à 1977, traduisant l'augmentation de la concurrence dans les peuplements les plus denses. La différence entre $E$ et $D$ est encore très faible $(2,9 \mathrm{~cm})$ et non significative.

Pour les circonférences dominantes, on observe la même tendance, mais il n'y a aucune différence significative entre traitements. 


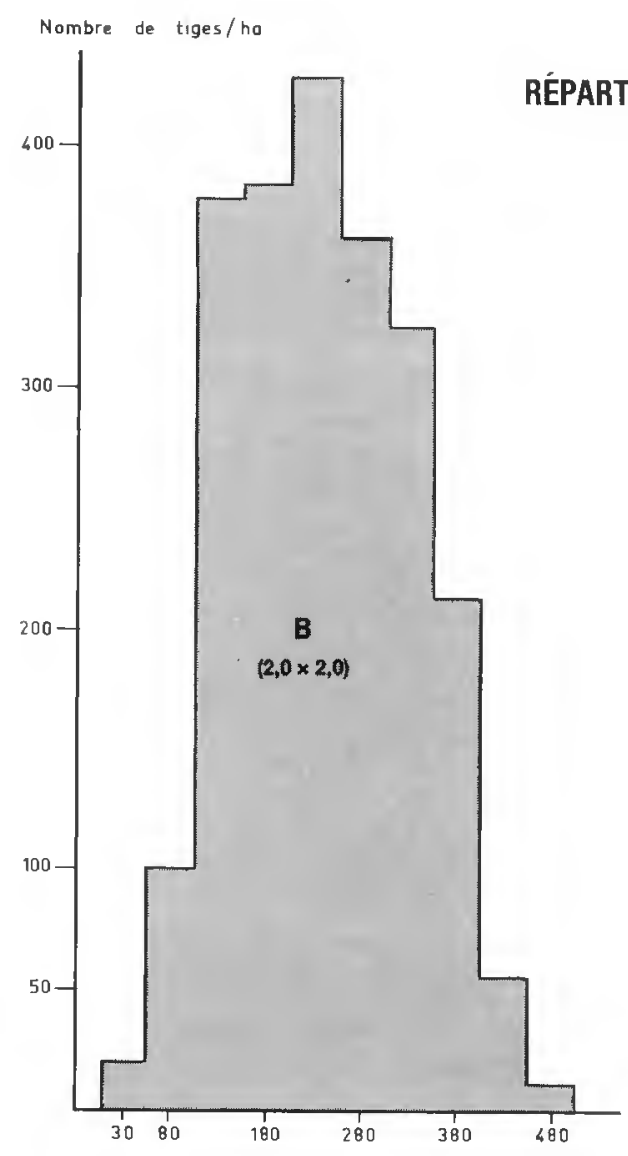

Figure 1
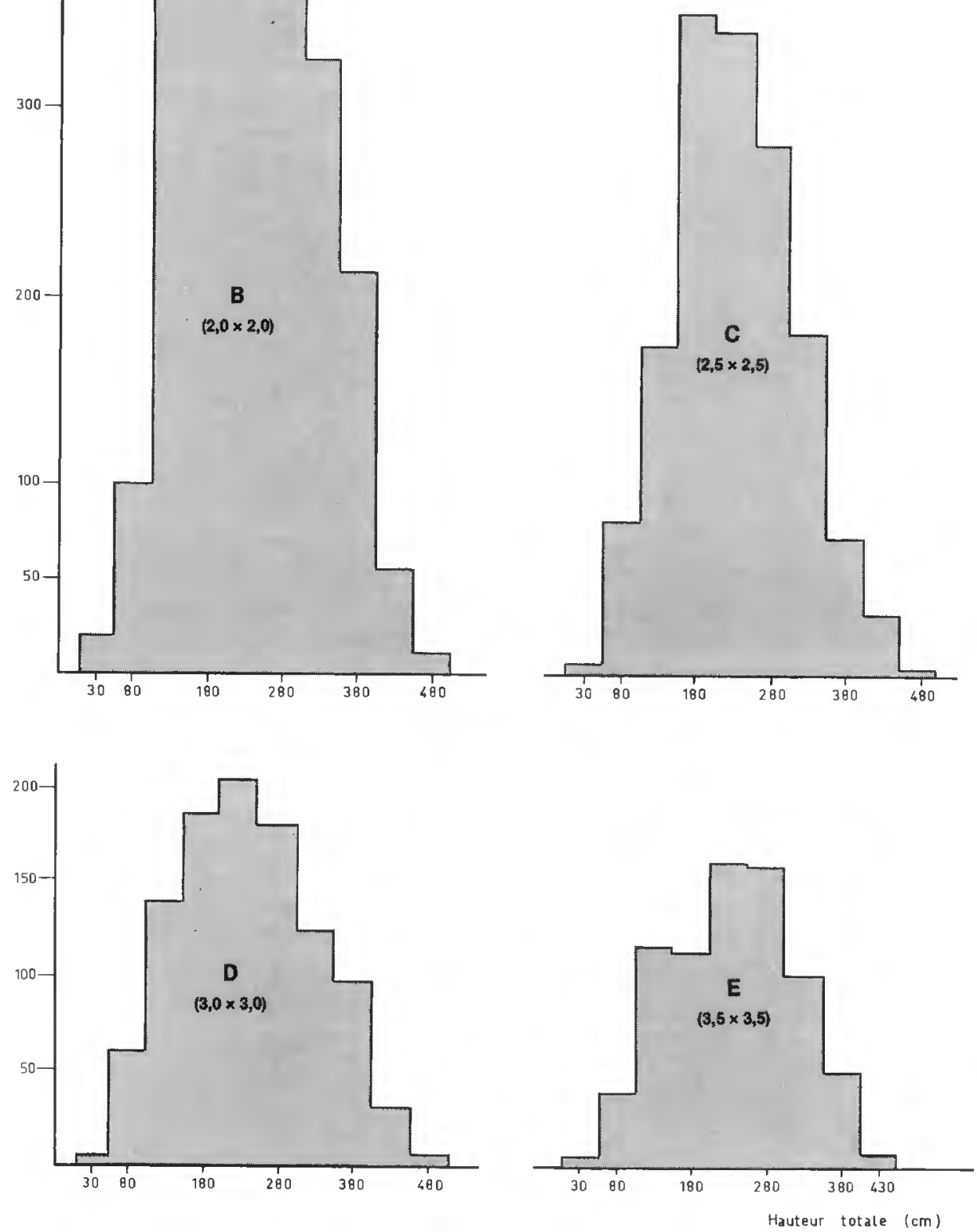


\section{H. OSWALD}

Il faut ici examiner la structure des peuplements par l'intermédiaire de la rẻpartition des tiges par classes de circonférence (figure 2). On constate, tout d'abord, que l'étendue totale des classes de circonférence est sensiblement la même, quel que soit l'espacement à la plantation. Ceci conduit à un aplatissement de plus en plus important quand la densité diminue.

D'autre part, seul le traitement B présente pour l'instant une légère dissymétrie à gauche de la courbe de distribution, signe d'une forte compétition entre arbres, tandis que le traitement $E$ accuse une légère dissymétrie à droite; il est encore proche d'une "croissance libre ".

\section{Figure 2}
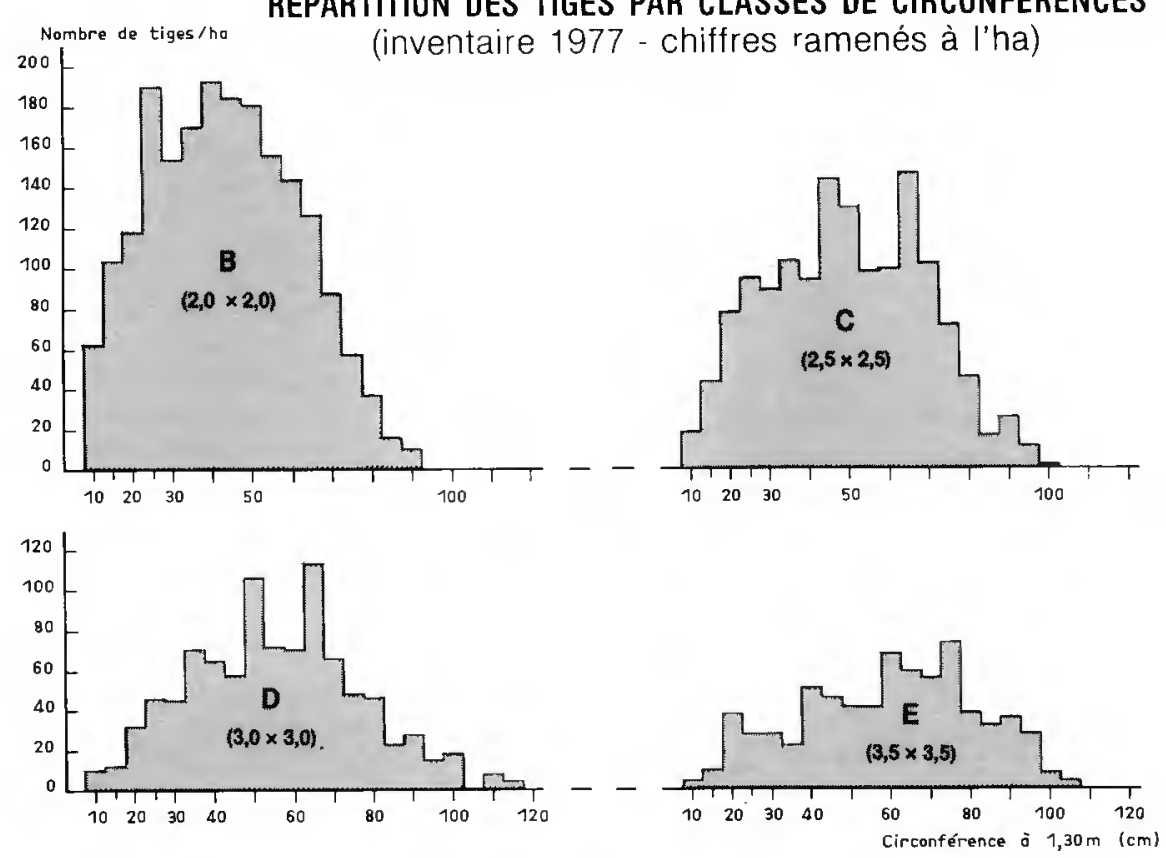

\section{Les surfaces terrières}

Les surfaces terrières augmentent fortement avec la densité; elles sont très différentes entre les densités extrêmes $\mathrm{E}$ et $\mathrm{B}(53 \%$ et $100 \%$ en $1974 ; 63 \%$ et $100 \%$ en 1977). On observe cependant, entre 1974 et 1977, un léger ralentissement de l'accroissement de la surface terrière du traitement $B$.

L'accroissement en surface terrière, entre 1974 et 1977, en valeur absolue est sensiblement le même dans les quatre traitements; on trouve le maximum dans le traitement $C\left(10,27 \mathrm{~m}^{2}\right)$ et le minimum $\left(8,61 \mathrm{~m}^{2}\right)$ dans le traitement $\mathrm{E}$.

On constate, à partir de 1977, une certaine coupure entre les traitements E-D d'une part et B-C d'autre part.

Si l'on regarde le taux d'accroissement en surface terrière, pour la même période, on trouve, comme on pouvait s'y attendre, une bonne liaison avec l'espacement. En fixant $B=100 \%$, on obtient: $\mathrm{C}=106 \%, \mathrm{D}=\mathbf{1 1 1} \%$, et $\mathrm{E}=118 \%$. 
On peut considérer que pour l'instant la croissance en surface terrière, au moins dans le traitement $E$, a été maximale et proche d'une "croissance libre" hors compétition.

\section{Le volume et la forme des tiges}

Il faut d'abord prèciser qu'il s'agit d'un volume commercial de la tige (VTT) qui est obtenu en mesurant la circonférence au milieu de la hauteur totale de la tige - cette dernière peut être mesurée avec une plus grande précision pour les arbres sur pied que la hauteur de découpe bois fort $(7 \mathrm{~cm}$ de diamètre) - et en faisant correspondre la section à un cylindre dont la longueur est la hauteur totale de l'arbre.

Les volumes sur pied ont été calculés à partir d'un tarif de la forme VTT $=a+b C^{2}$ établi sur un échantillon représentatif.

Les volumes totaux tiges des quatre traitements sont encore davantage liés à la densité que les surfaces terrières et on constate une perte encore considérable de production, qui atteint $50 \%$ dans le traitement $E(B=100 \%, C=79 \%, D=65 \%, E=50 \%)$. C'est là un résultat très important, au moment où l'on parle de "plantations énergétiques " qui seraient récoltées à courte révo. lution.

Si I'on considère le volume de l'arbre moyen la situation s'inverse, mais les différences sont moins prononcées $(E, D=B, C)$. En valeur relative, on obtient : $B=100 \%, C=109 \%, D=$ $135 \%, E=134 \%$.

En ce qui concerne le coefficient de forme du peuplement $F=V T T /\left(G \times H_{g}\right)$, on trouve, comme il fallait s'y attendre, une diminution constante de ce dernier lorsque l'espacement augmente. En valeur relative, avec $\mathrm{B}=100 \%$, on obtient $\mathrm{C}=91 \%, \mathrm{D}=87 \%$ et $\mathrm{E}=80 \%$.

Cette " dégradation" de la forme des tiges dans les larges espacements entraine une diminution non négligeable du rendement en sciages qui n'est pas compensée par une plus grande circonférence.

Plantation d'Abies grandis; arboretum de la Sivrite (54) Photo CANTA - CNRF

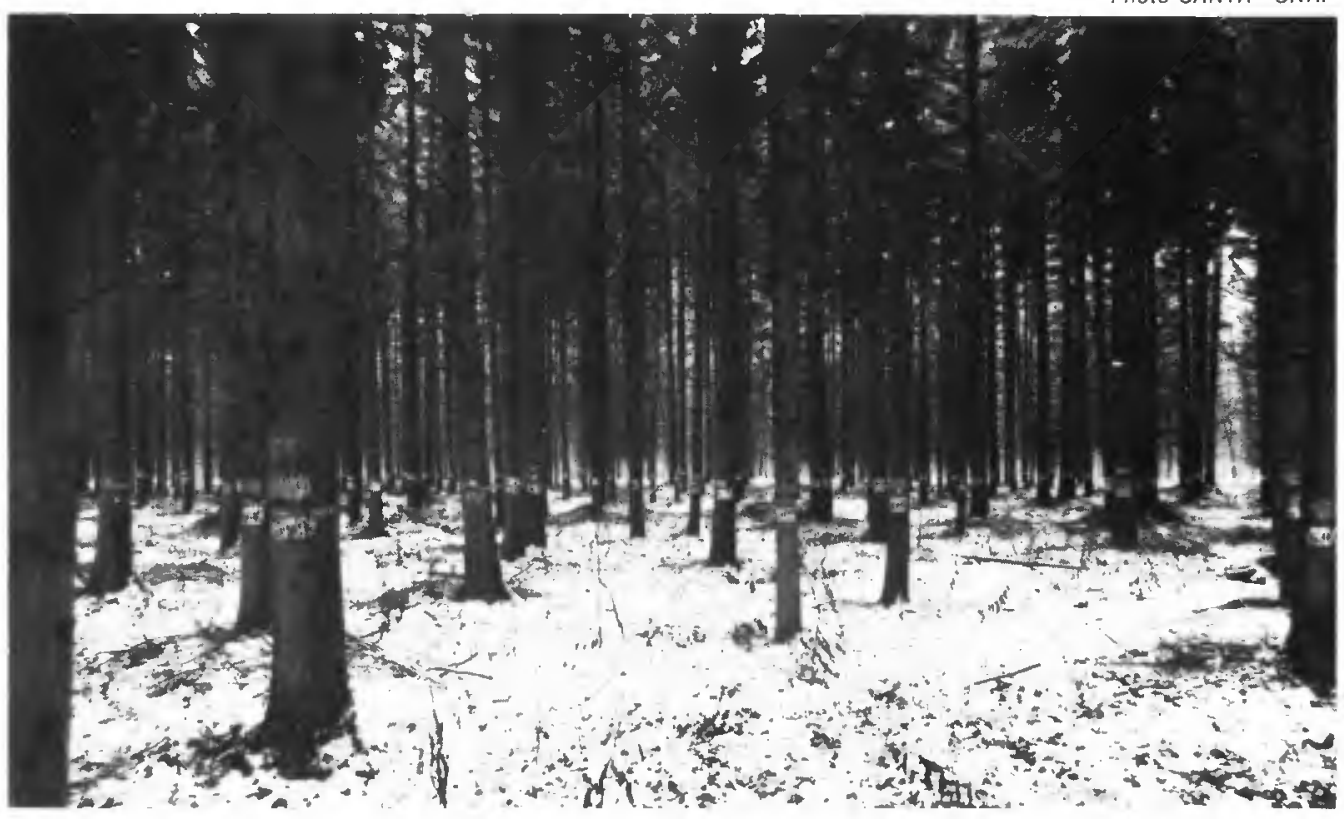




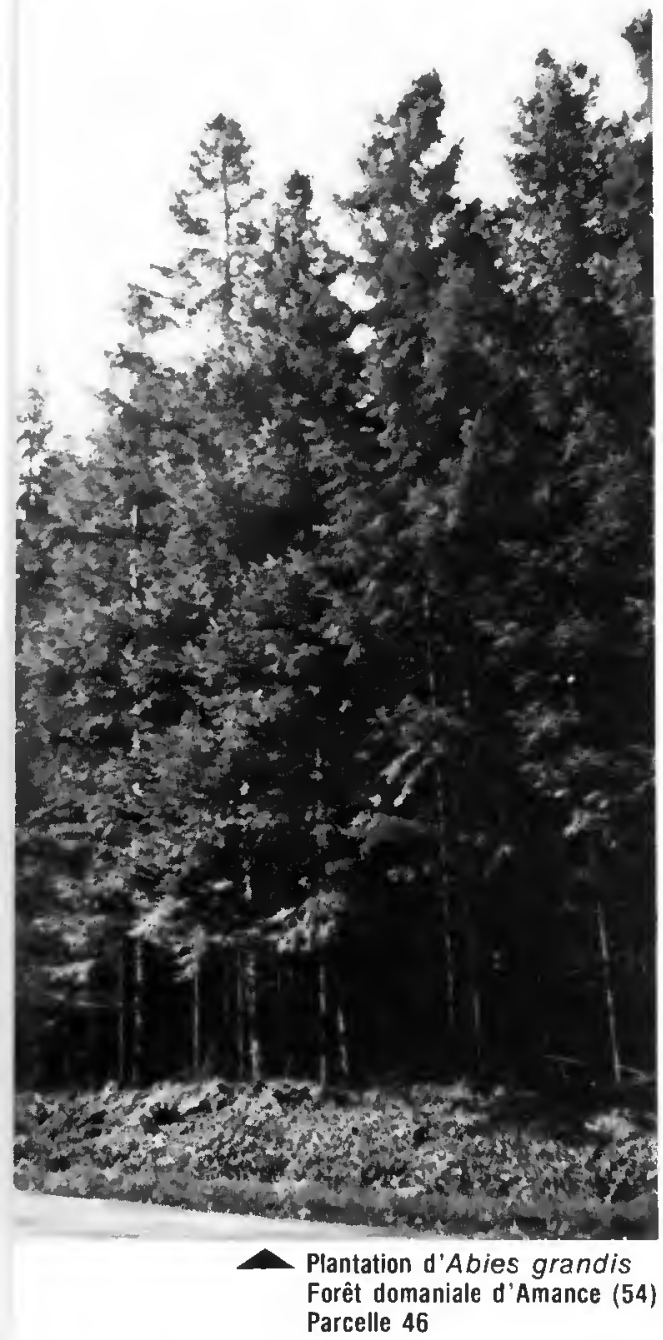

Figure 3

\section{HAUTEUR DOMINANTE ET PRODUCTION TOTALE EN FONCTION DE L'AGE COMPARÉES A LA TABLE DE PRODUCTION ANGLAISE (abies grandis) - Yield (classes 18 à 26)}

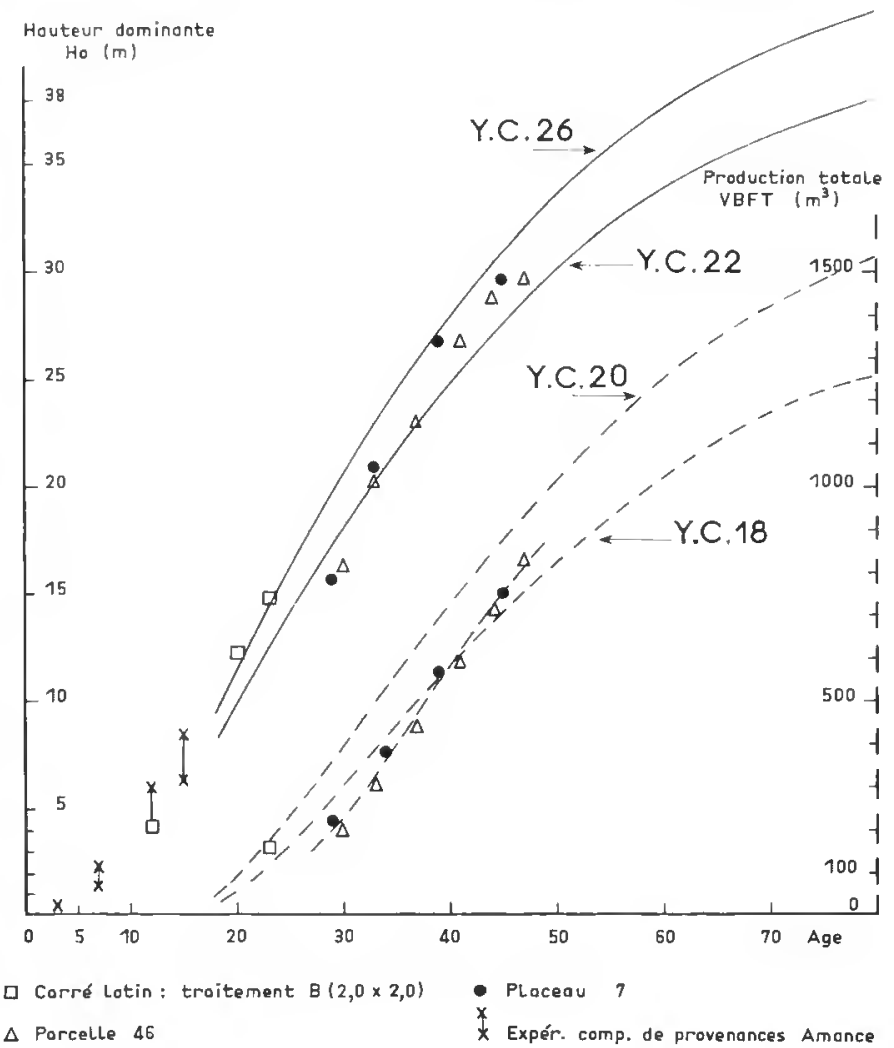

Parmi les coefficients de forme, il faut également mentionner le rapport H/D (1), qui certes exprime la forme de la tige, mais qui est également ètroitement lié à l'importance relative du houppier, à la position sociologique de l'arbre et sa vigueur, ainsi qu'à sa stabilité vis-à-vis de la neige et du vent (Abetz, 1976),

Dans la figure 3, nous avons présenté le rapport H/D sous forme d'abaque, en y faisant figurer les courbes moyennes de hauteur pour les traitements $E$ et $B$, ainsi que la courbe de hauteur d'un peuplement d'Abies grandis de 47 ans (Parcelle 46, en forêt domaniale d'Amance).

Des auteurs allemands cités par Abetz ont montré que les arbres dominants et codominants ont souvent des rapports H/D inférieurs à 100 et on préconise pour le choix des " arbres d'avenir " des rapports inférieurs à 80 ; l'avantage principal d'un classement sociologique à partir des rapports $H / D$ réside dans le fait qu'il est basé sur des mesures objectives et relativement faciles à obtenir.

Les rapports H/D des arbres moyens et dominants en 1974 et en 1977 sont, comme on pouvait s'y attendre, liés à l'espacement. On ne constate encore qu'une faible différenciation sociale dans le traitement $\mathrm{E}$ par rapport au traitement B (figure 3). 


\section{Quelques données complémentaires}

Une étude approfondie de la bibliographie sur cette essence fait apparaître une grande pauvreté de données dendrométriques et sylvicoles, surtout dans son aire d'origine, mais également dans les pays où elle a été introduite. La seule référence européenne, d'une certaine importance, est la table de production anglaise (Hamilton et Christie, 1971); nous l'utiliserons à titre de comparaison, de même que quelques résultats de plantations comparatives de provenances françaises et de quelques peuplements adultes.

Les essais français de plantations comparatives de provenances.

Les résultats des expériences françaises installées dans des stations très variées entre 1964 et 1966 (8 dispositifs) viennent d'être publiés (Hermann et Birot, 1978 ; Noël, 1979). Elles confirment la supériorité des provenances de la région de l'lle de Vancouver et la très bonne place des provenances artificielles françaises.

La provenance artificielle "Pezanin ", utilisée dans notre expérience d'espacement, est également présente dans plusieurs dispositifs de la station d'Amélioration des arbres forestiers de l'Institut national de la Recherche agronomique, et notamment en forêt domaniale d'Amance, dans les mêmes conditions climatique et édaphique. L'espacement est de $2,0 \mathrm{~m} \times 2,0 \mathrm{~m}$ et correspond donc à notre traitement B. Un léger abri de 80 brins/ha a été conservé pendant 2 ans.

Les hauteurs moyennes et dominantes (moyenne des 50 plus hauts arbres à l'hectare) à l'âge 3, 7 12 et 15 ans nous ont permis de retracer la croissance juvénile de la provenance. La hauteur moyenne à 12 ans et le coefficient de variation sont sensiblement les mèmes dans les deux dispositifs (figure 4); la mortalité à 15 ans $(6,9 \%)$ est légèrement inférieure dans l'essai de provenances.

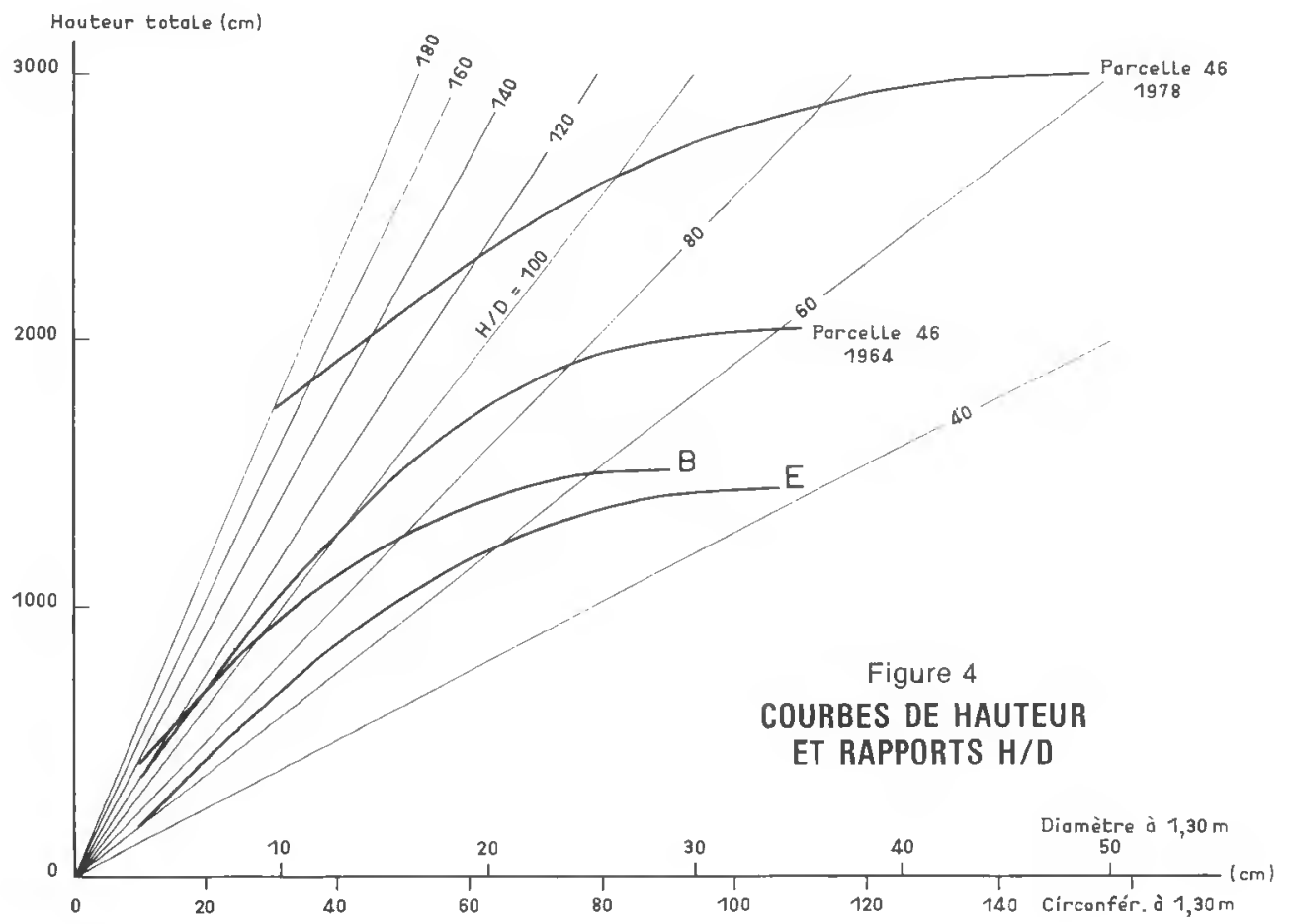


H. OSWALD

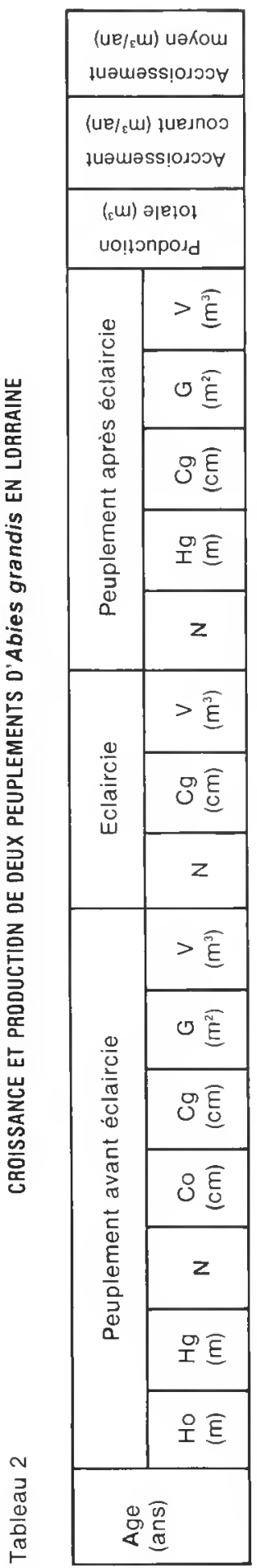

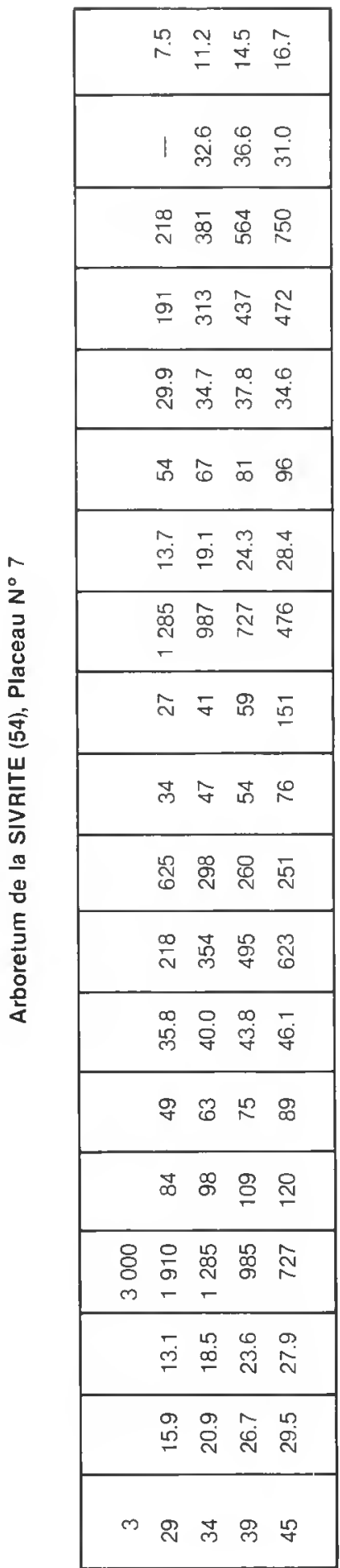

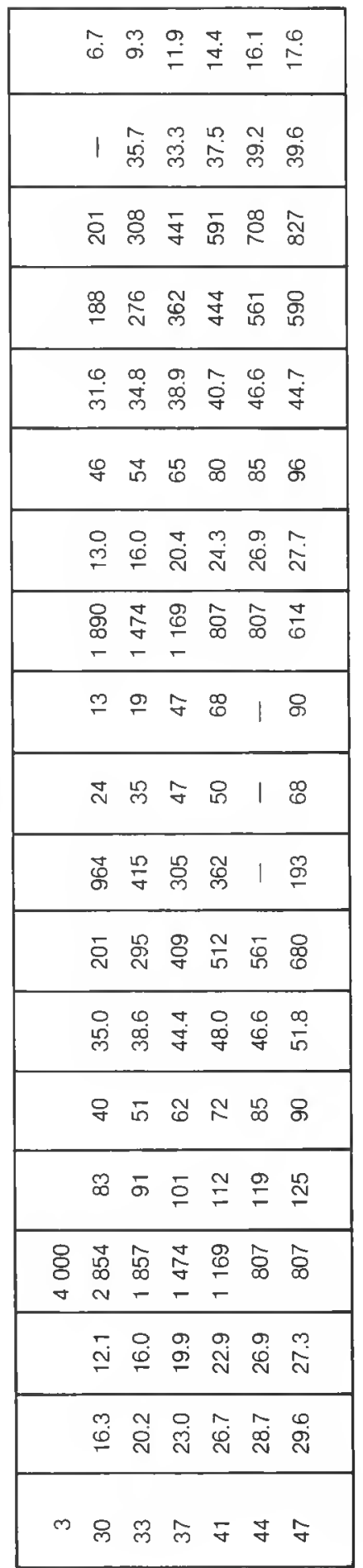



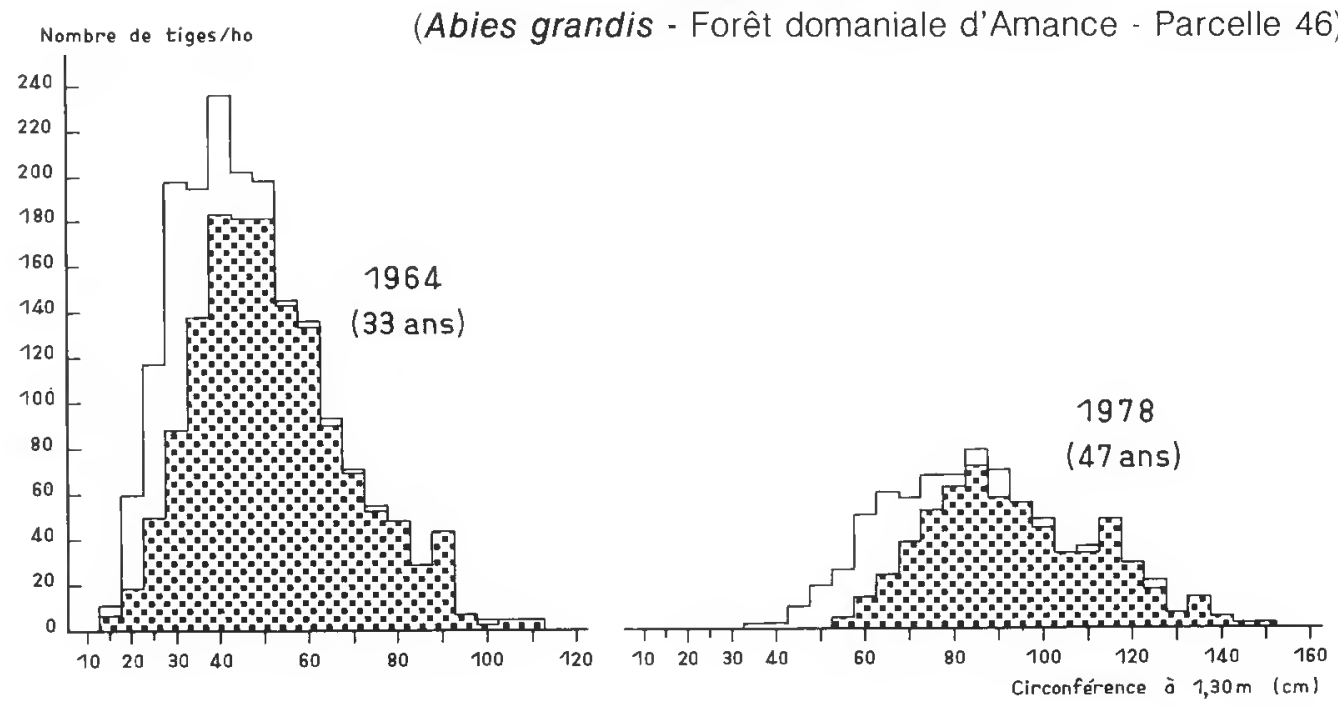

Peuplemen/s adultes

Nous possèdons dans la région deux peuplements de même âge (46 ans en 1977) : l'un (placeau 7) se trouve dans l'arboretum de la Sivrite, jouxtant la forêt domaniale de Haye (54), l'autre (parcelle 46) en forêt domaniale d'Amance. Les deux placettes sont éclaircies et mesurées régulièrement par la station de Sylviculture et de Production du Centre national de Recherches forestières. Les deux peuplements sont restés pendant au moins 15 ans sous un abri latéral et/ou un abri vertical.

Les résultats des mesures dendrométriques successives sont présentés dans le tableau 2. Pour la placette de la parcelle 46 , nous avons également présenté les courbes de hauteurs aux âges de 33 et 47 ans (figure 3) et les répartitions des tiges par classes de circonférences avant et après éclaircie aux mêmes âges (figure 5).

\section{Comparaison avec la table de production anglaise}

La table de production anglaise pour Abies grandis constitue la seule source d'information pour la croissance et la production de cette essence, notamment en Europe. Les principes de sa construction et son mode d'emploi ont été décrits par Martinot-Lagarde et Bartet (1975), mais nous citerons les particularités essentielles.

- L'âge du peuplement est défini, comme le nombre de saisons de végétation, depuis la plantation (dans nos graphiques et tableaux, nous avons cependant utilisé l'âge physique des peuplements).

- Les classes de production générales sont définies par l'accroissement moyen annuel maximum, ces accroissements sont espacés de 2 en $2 \mathrm{~m}^{3} / \mathrm{ha} / \mathrm{an}$. Elles sont présentées par des courbes hauteur dominante/âge plus faciles à utiliser. 


\section{H. OSWALD}

- Il existe une liaison unique entre la production totale bois fort tige et la hauteur dominante. Pour tenir compte d'éventuelles variations locales, on introduit la notion de "niveau de production ". On distingue généralement trois niveaux $(a, b, c)$; le niveau moyen " $b$ " est celui de la classe de production générale, le niveau supérieur " a " ou inférieur " c " est obtenu simplement en utilisant la production de la classe de production immédiatement supérieure ou inférieure.

- Le nombre de tiges après éclaircie peut être représenté en fonction de la hauteur dominante par une courbe unique, quelle que soit la classe de production. L'éclaircie pratiquée peut ètre considérée comme une éclaircie très forte à ne pas dèpasser.

\section{La croissance en hauteur}

Dans la figure 4, nous avons présenté l'évolution de la hauteur dominante, en fonction de l'âge physique, pour les classes de production Y C 22 et Y C 26. Nous y faisons également figurer les données de nos dispositifs expérimentaux cités précédemment.

La croissance en hauteur de nos placettes est assez bien encadrée par ces deux courbes. Les hauteurs plus faibles entre 29 et 35 ans de nos deux placettes d'éclaircies peuvent partiellement être imputées à un abri trop dense pendant les vingt premières années (Lacaze et Pardé, 1963).

Röhrig (1978), qui analyse les différents résultats d'introduction de Abies grandis en Allemagne, arrive aux mêmes constatations. Stage (1963), dans la construction de courbes de "site index" polymorphes, tient compte d'un éventuel ralentissement de la croissance en hauteur dans le jeune âge dû notamment à l'existence d'un couvert prolongè.

II semble tout à fait possible que nos placettes atteignent une hauteur dominante de plus de $40 \mathrm{~m}$, à l'âge de 80 ans, comme le montrent quelques bouquets dans l'arboretum d'Amance et à l'ètang de Brin dans la même forèt.

D'une maniére générale, on constate pour la plupart des essences en Grande-Bretagne que la croissance en hauteur démarre plus rapidement, mais elle ralentit aussi plus tôt. L'éventail des hauteurs dominantes à 83 ans s'étend de 30 à $45 \mathrm{~m}$, d'après la table anglaise.

\section{La production en volume}

Dans la figure 4, nous faisons également figurer la production totale en bois fort tige des classes de production 18 et 22, ainsi que les données de nos placettes.

II faut d'abord mentionner que la production totale pour une même hauteur dominante donnée est nettement plus élevée en Grande-Bretagne qu'en Europe centrale, probablement à cause d'une période de végétation plus longue et l'absence de déficits hydriques.

Les données de nos placettes suggèrent aussi une allure de l'évolution de la production totale légèrement différente que celle présentée dans la table. Tout laisse cependant penser, qu'au moment de la culmination de l'accroissement moyen vers 58 ans, nous atteindrons la classe 20 et à la fin de la révolution, vers 80 ans, la production moyenne par hectare et par an sera de 18 à $\left.19 \mathrm{~m}^{3}{ }^{2}\right)$.

(1) $H=$ hauteur totale en $\mathrm{cm}, \mathrm{D}=$ diamétre à $1,30 \mathrm{~m}$ en $\mathrm{cm}$.

(2) A titre de référence : l'accroissement moyen annuel maximum des places d'expérience de chẻne que nous suivons dans les mémes forèts sur stations comparables se situe entre 5 et $6 \mathrm{~m}^{3} / \mathrm{ha} / \mathrm{an}$, atteints a 120 et 130 ans seulement. 
II est fort probable que dans le nord et nord-ouest de la France, la table anglaise s'applique avec plus de rigueur, comme il a été montré pour d'autres essences (Le Goff, 1974).

Les eclaircies

L'objectif des tables anglaises est la production maximum en volume et les prélèvements par l'éclaircie correspondent à environ $70 \%$ de l'accroissement moyen maximum par an jusqu'à la moitié de la révolution, ils diminuent ensuite. Cette intensité est appelée "l'intensité marginale ", c'est-à-dire qu'elle n'entraîne pas encore des pertes de production. La nature de l'éclaircie est mixte. La rotation peut être variable, si on respecte l'intensité maximum.

Dans la figure 6, nous présentons le nombre de tiges, après éclaircie, en fonction de la hauteur dominante pour l'ensemble de nos dispositifs, ainsi que la courbe anglaise pour la classe de production 22 qui, comme nous venons de le dire, est quasiment unique pour toutes les classes de production.

Figure 6

\section{NOMBRE DE TIGES APRĖS ÉCLAIRCIE EN FONCTION DE LA HAUTEUR DOMINANTE}

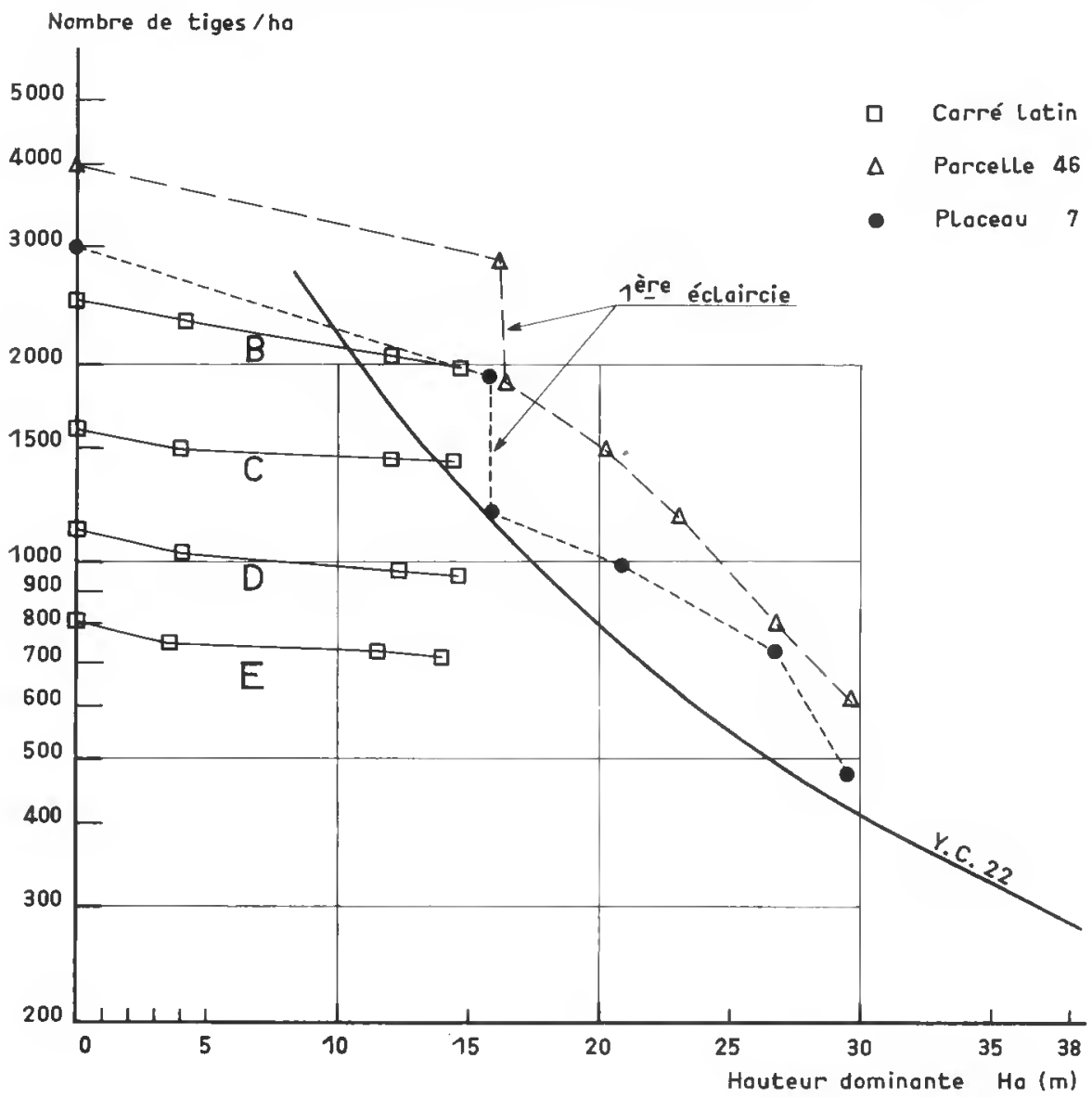




\section{H. OSWALD}

On remarque tout d'abord que nos deux placettes d'éclaircies ont des densités plus élevées que "la norme anglaise"; mais nous pensons arriver progressivement à une densité finale de 300 tiges à l'hectare pour une hauteur dominante de $40 \mathrm{~m}$, densité légèrement plus élevée que celle conseillée par la table.

En ce qui concerne les densités du dispositif d'espacement, on observe seulement la mortalité naturelle. Dans le traitement $E(3,5 \times 3,5 \mathrm{~m})$, on peut estimer que la première éclaircie n'interviendra seulement que vers une hauteur dominante supérieure à $25 \mathrm{~m}$, c'est-à-dire entre 40 et 45 ans ; le traitement $B$ est à éclaircir dès maintenant.

\section{DISCUSSION DES RÉSULTATS ET COMPARAISON AVEC L'EXPÉRIENCE D'ESPACEMENT DE DOUGLAS}

L'absence de données dendrométriques sur Abies grandis, notamment, en ce qui concerne l'influence de l'espacement à la plantation sur l'évolution des peuplements, nous incite à tenter une comparaison avec une expérience d'espacement de Douglas (Pseudotsuga menziesii Mirb.) installée dans la même parcelle deux ans plus tôt. Les résultats successifs de cette expérience ont été publiés par Bartoli et Decourt 1971, Bartoli 1971, Oswald et Pardé 1976.

Nous ne reviendrons pas en détail sur ces résultats et nous n'insisterons pas non plus sur les effets généraux des espacements qui ont été abondamment analysés par Hamilton et Christie 1974, et Low et Van Tol 1974.

Rappelons d'abord que la comparaison porte essentiellement sur les traitements $B(2,0 \times 2,0 \mathrm{~m})$, $C(2,5 \times 2,5)$ et $D(3,0 \times 3,0 \mathrm{~m})$, que l'âge de la comparaison est de 21 ans pour le Douglas et de 23 ans pour $A$. grandis, mais chaque fois qu'il sera possible, nous ferons l'interpolation pour ramener à un même âge de comparaison (21 ans). Autre remarque importante : la plantation de Douglas est restée seulement 5 ans au lieu de 7 ans sous abri qui, du reste, ètait moitié moins dense que celui d'A. grandis.

On observe les différences suivantes:

- La mortalité naturelle est globalement de moitié chez le Douglas,

- A. grandis, comme beaucoup de sapins, a une croissance juvénile en hauteur plus lente. La différence des hauteurs dominantes à 21 ans (interpolée pour A. grandis) est de $3,0 \mathrm{~m}$; elle est partiellement due au couvert.

- La variance des circonférences et des hauteurs est plus grande chez A. grandis; elle sem. ble aussi moins liée à l'espacement.

- On observe chez A. grandis une tendance plus marquée à une diminution de la hauteur dominante dans les très larges espacements (traitement E); cet effet n'est cependant pas significatif.

- Les circonférences dominantes chez le Douglas sont significativement liées à l'espacement $(A \neq B, C \neq D)$; chez $A$. grandis, nous trouvons sensiblement les mêmes valeurs pour les traitements $B, C$ et $D$ avec un décrochement de $E$, mais les différences entre traitements ne sont pas significatives.

- Les surfaces terrières chez A. grandis sont inférieures environ d'un tiers à celles du Douglas (interpolation à 21 ans), ce qui s'explique seulement, pour moitié, par la mortalité plus faible chez le Douglas, car les circonférences moyennes restent inférieures de $10 \%$ (B) à $16 \%$ (D) chez A. grandis, malgré la plus forte mortalité. 
- En ce qui concerne les volumes bois tige total (VTT), nous ne pouvons pas faire d'interpolation. Du fait que la surface terrière, la hauteur, mais également le coefficient de forme du peuplement sont globalement supérieurs chez le Douglas, les volumes le sont encore davantage. Nous nous intéresserons plus particulièrement aux valeurs relatives des traitements présentées dans le tableau 3 cidessous:

Tableau 3

\begin{tabular}{|l|c|c|c|c|c|}
\hline Essence & $\begin{array}{c}\text { A } \\
1,5 \times 1,5\end{array}$ & $\begin{array}{c}\text { B } \\
2,0 \times 2,0\end{array}$ & $\begin{array}{c}\text { C } \\
2,5 \times 2,5\end{array}$ & $\begin{array}{c}\text { D } \\
3,0 \times 3,0\end{array}$ & $\begin{array}{c}\text { E } \\
3,5 \times 3,5\end{array}$ \\
\hline Douglas 21 ans & $111 \%$ & $100 \%$ & $81 \%$ & $74 \%$ & - \\
\hline A. grandis 23 ans & - & $100 \%$ & $79 \%$ & $65 \%$ & $50 \%$ \\
\hline
\end{tabular}

Les volumes diminuent donc plus rapidement avec la densité chez A. grandis ; ces pertes de production dans les espacements très larges ne pourront probablement pas être réduites notablement durant une révolution relativement courte de 60 ans.

Ceci conduit à préconiser des densités de plantation plus élevées chez $A$. grandis, quel que soit l'objectif de production. On peut penser qu'une densité de 1500 à 1800 plants à l'hectare sera suffisante.

\section{AVANTAGES ET INCONVÉNIENTS DE L'ABIES GRANDIS}

Sur le plan de la productivité, Aldhous et Low (1974) en Grande-Bretagne ont montré, sur des stations comparables, la supériorité d'A. grandis vis-à-vis de tous les autres résineux autochtones et introduits.

A. grandis peut être planté sur une large gamme de stations; de plus, l'interaction station x provenance s'est avérée assez faible (Noël 1979). Les productions les plus élevées sont cependant obtenues sur des sols riches et profonds, dans des régions à précipitations ou à état hygrométrique élevé (Aldhous et Low 1974, Pourtet 1964, Delvaux 1969).

Sa résistance vis-à-vis du froid est remarquable, mais il supporte également très bien des périodes de sécheresse estivale. Des provenances à débourrement plus précoce craignent les gelées tardives (Larsen J. Bo, 1978; Schwerin I. 1978). A. grandis supporte assez bien un couvert vertical ou latéral, mais sa vigueur est alors diminuée.

Sa fructification est régulière et abondante même en dehors de son aire naturelle et sa régénération naturelle peut être obtenue assez facilement.

Sa résistance vis-à-vis des facteurs biotiques dans son aire artificielle en Europe est moins bonne que celle du Douglas; mais aucun dépérissement massif n'est encore apparu. On a cependant signalé des attaques très localisées de Fomes annosus et d'Armillaria mellea. Parmi les insectes, il faut citer Adelges piceae qui a causé quelques dégâts en Grande-Bretagne (Aldhous et Low 1974) et aux États-Unis dans son aire d'origine (Hermann 1978). 


\section{H. OSWALD}

Fesia d considérer la qualité iechnologique du bois et son utilisátion. De nombreuses études ont montré que le bois d'A. grandis est caractérisé par une faible densité et dẹs propriétés mécaniques peu élevées notamment par rapport au Douglas. Ceci interdit souvent son emploi comme bois de construction de qualité. II peut être utilisé pour la fabrication de pâtes mécaniques et chimiques blanchies où il tient la comparaison avec d'autres essences (Epicéa de Sitka, etc.). Ceci tient essentiellement à la bonne longueur des fibres qui atteignent en moyenne $3 \mathrm{~mm}$ dans les cernes annuels âgés de 10 ans et plus, et à un rendement associé en pâte de l'ordre de 45 à $50 \%$ (Janin et Nepveu 1979).

Des essais de déroulage ont également été concluants (Centre technique du Bois 1975) et ouvrent une nouvelle voie d'utilisation.

On trouve aussi des provenances à infradensité plus élevée (Polge 1968), mais cette qualité s'accompagne d'une perte de vigueur non négligeable (Noël 1979). L'amélioration génétique pourra peut-être offrir à long terme une solution à ce problème.

D'autre part, on constate qu'une diminution de la largeur des cernes augmente les qualités mécaniques du bois (Polge 1963, Sacré 1978); on peut alors se demander si le maintien du peuplement à une densité assez élevée tout au long de la révolution ne permettrait pas à la fois une amélioration des propriétés mécaniques (au moins pour les arbres dont la circonférence est au plus égale à la moyenne) et une production maintenue à son niveau maximal. Le dispositif expérimental permettrait ultérieurement de vérifier cette hypothèse.

Malgré les inconvénients que nous venons d'évoquer, on constate á nouveau, notamment en République fédérale d'Allemagne, un regain d'intérêt pour cette essence.

Une importante expérience d'introduciion d'Abies grandis et de Douglas est en préparation avec plus de 40 implantations dont environ 30 en Basse-Saxe (Gussone 1978). Le dispositif expérimental utilise une seule provenance pour chaque essence, il comporte trois traitements (A. grandis pur, Douglas pur et un mélange des deux) avec trois répétitions. La densité de plantation est de 3077 plants à l'hectare $(2,5 \mathrm{~m} \times 1,3 \mathrm{~m})$.

D'autre part, une expérience internationale de comparaison de provenances d'Abies grandis est en cours de réalisation dans le cadre de I'I.U.F.R.O. (Union internationale des instituts de recherches forestières).

II serait très souhaitable, qu'en France également, on s'intéresse davantage à A. grandis, notamment sur le plan de la production, de la conduite des peuplements et des qualités technologiques du bois. L'intérêt de $A$. grandis se situe à deux niveaux, d'une part dans une perspective de production de "bois matière première renouvelable" grâce à sa productivité très élevée, et d'autre part comme une alternative au Douglas en assurant en peuplement pur ou en mélange une plus grande diversité et une meilleure stabilité écologique.

Avec la collaboration technique de A. Tisserand
Helfried OSWALD

Chargé de Recherches

Station de Sylviculture et de Production Centre National de Recherches Forestieres (I.N.R.A.)

Champenoux 54280 SEICHAMPS 


\section{BIBLIOGRAPHIE}

ABETZ (P.). - Beiträge zum Baumwachstum. Der h/d Wert-mehr als ein Schlankheitsgrad. Forst- u. Holzw. 31 (19), 1976, p. 389-393.

ALDHOUS (J.R.) et LOW (A.J.). - The potential of Western Hemlock, Western Red Cedar, Grand Fir and Noble Fir in Britain. Forestry Commission Bulletin n 49,1974105 p. H.M.S.O.

BARTOLI (M.). - Premiers résultats à 11 et 15 ans d'une expérience de densité sur le douglas. Revue forestière trançaise $n^{\circ} 6,1971$, p. 605-608.

BARTOLI (M.) et DECOURT (N.). - Densité de plantation. Ėtude bibliographique et premiers résultats d'une expérience sur le douglas. Annales Sciences forestières, 1971, 28 (1), p. 59-81.

CENTRE TECHNIQUE DU BOIS - Aptitude au déroulage et à la fabrication de panneaux de contreplaqué, du bois d'Abies grandis provenant des reboisements trançais. Centre technique du Bois, Paris, 1975,12 p.

DELVAUX (J.). - Le choix objectif de l'essence en sylviculture. Comportement relatif d'Abies grandis LDL et de Pseudotsuga menziesii viridis Franco en Belgique. Bulletin Societe Royale forestiere de Belgique, 76 (11) 1969 p. 445-459.

GUSSONE (H.-A.). - Der waldbauliche Abies Grandis-Anbauversuch 1980. in : Grundlagen für den Anbau von Abies Grandis. Schriften aus der Forstlichen Fakultăt Göttingen u.d. Niedersáchsischen Forstlichen Versuchsanstalt. J.D. Sauerländer's Verlag, Frankfurt a.M. vol. 54, 1978, p. 83-90.

HAMILTON (G.J.) et CHRISTIE (J., M.). - Forest Management tables (metric). Forestry Commission Booklet $n^{\circ} 34$, 1971, 201 p. Her Majesty's stationery office London.

HAMILTON (G., J.) et CHRISTIE (J., M.). - Influence of spacing on crop caracteristics and yield. Forestry Commission Bulletin $n^{\circ} 52,1974,92$ p. Her Majesty's stationery office London.

HERMANN (R., K.). - Abies grandis in ihrem Heimatland; in : Grundlagen für den Anbau von Abies grandis. Schriften aus der forstl. Fakultăt der Universităt Gottingen u. der Niedersăchsischen forstl. Versuchsanstalt. J.D. Sauerländer's Verlag Frankfurt a.M. Vol. 54, 1978, p. 7-24.

HERMANN (R., K.) et BIROT (Y.). - Vorläufige Ergebnisse des ersten französischen Provenienzversuches mit Abies Grandis; in : Grundlagen für den Anbau von Ables grandis. Schriften aus der forstl. Fakultat der Universităt Göttingen $u$. der Niedersăchsischen forstl. Versuchsanstalt. J.D. Sauerländer's Verlag Frankfurt a.M. Vol. 54, 1978, p. 67-82.

JANIN (G.) et NEPVEU (G.). - La qualité des bois. Institut national de la Recherche Agronomique - Centre national de Recherches forestières, Station de recherches sur la Qualité des Bois. Manuscrit, $1979,15 \mathrm{p}$.

LACAZE (J.F.) et PARDÉ (J.). - Les enseignements de l'arboretum de la Sivrite. Revue forestière française $n^{\circ} 2,1963$, p. 92-115.

LACAZE (J.F.) et TOMASSONE (R.). - Contribution à l'étude de la variabilité infraspécifique d'Abies grandis (Lindl). Caractéristiques juvéniles. Annales des Sciences forestières, 24 (4), 1967, p. 277-326.

LARSEN (J.) - Ergebnisse von Frost- und Trockenresistenz-Untersuchungen an Abies grandis. In : Grundlagen für den Anbau von Abies grandis. Schriften aus der forstl. Fakultăt der Universităt Gsttingen u. der Niedersächsischen forstl. Versuchsanstalt. J.D. Sauerländer's Verlag Frankturt a.M. Vol. 54, 1978, p. 7-24.

LE GOFF (N.). - La croissance du hêtre en France : utilisation des tables de production étrangères pour suivre l'évolution des peuplements. Institut national de la Recherche Agronomique - Centre national de Recherches forestières, Station de Sylviculture et de Production. Doc. $n^{\circ} 3,1974,73 \mathrm{p}$.

LOW (A.J.) et VAN TOL (G.). - Initial spacing in relation to stand establishment. In : Proceedings Symposium stand establishment (IUFRO) Wageningen, P.B. oct. 1974, p. 296-319.

MARTINOT-LAGARDE (P.) et BARTET (P.). - Les tables de production britanniques. Office national des forêts Section technique. Doc. $n^{\circ} 2,1975,45$.

NOEL (J.C.). - Croissance et qualité du bois d'Abies grandis (Lindl.); Étude de la variabilité inter et intra populations. Station d'amélioration des arbres forestiers. Institut national de la Recherche agronomique Centre de Recherches forestières d'Orléans. Doc $n^{\circ} 2,1979,87 \mathrm{p}$.

OSWALD (H.) et PARDÉ (J.). - Une expérience d'espacement de Douglas en forêt domaniale d'Amance. Revue forestiére trançaise $n^{\circ} 3,1976$, p. 185-192.

PARDÉ (J.). - Contribution à l'étude de la productivité des reboisements de la région du plateau de Millevaches. Revue forestiere trançaises 17 (8-9), 1960, p. 557-571.

PARDÉ (J.) et PRAX (J.L.). - Les résineux dans le nord-ouest de l'Hérault. Revue forestiere françaises 21 (4), 1964, p. 286-301.

POLGE (H.). - Compte rendu des études technologiques taites sur quatre essences en provenance de l'arboretum de la Sivrite. Revue forestiere française $n^{\circ} 2,1963$, p. 116-126.

POLGE $(\mathrm{H}$.). - Tests précoces de la qualité du bois sur 25 provenances d'Ables grandis. Annales des Sciences forestières 25 (1), 1968, p. 3-23. 


\section{H. OSWALD}

POURTET (J.). - Les repeuplements artificiels. Ėcole nationale du Génie rural, des Eaux et des forêts Nancy $3^{\circ}$ édition, 1964, $278 \mathrm{p}$.

RÖHRIG (E.). - Anbauergebnisse mit Abies grandis in Deutschland in : Grundlagen für den Anbau von Abies grandis. Schriflen aus der forstl. Fakulläl der Universităt Göllingen u. der Niedersächsischen forstl. Versuchsansıall. J.D. Sauerländer's Verlag Frankfurt a.M. Vol. 54, 1978, p. 37-52.

SACRÉ (E.). - Étude physique et mécanique du bois de Sapin de Vancouver, Abies grandis (Dougl.) Lindl. et de Tsuga de Californie, Tsuga heterophylla (Raf.) Sarg. de provenance belge. Bulletin de la Sociélé royale forestière de Belgique. 85 (4), 1978, p. 141-157.

SCHWERIN (1.). - Wachstum und Austrieb von 20 Abies grandis Herkünften. in : Grundlagen für den Anbau von Abies grandis. Schriften aus der Forstlichen Fakultät Gölingen $u$. d. Niedersächsischen Forstlichen Versuchsanstall. J.D. Sauerländer's Verlag, Frankfurt a.M. Vol. 54, 1978, p. 31-36.

STAGE (A.R.). - A mathematical approach to polymorphic site index curves for grand fir. Forest Science 9 (2), 1963, p. $167-180$.

\section{A NOS ABONNÉS}

Les abonnements à la Revue forestière française sont renouvelables avec le numéro 1 ou le numéro 4 de chaque année. Nous faisons désormais paraître cette indication sur nos pochettes d'envoi, à droite de votre nom.

\begin{tabular}{|c|c|}
\hline Exemple & $\begin{array}{l}\text { M. DUBOIS Pierre } \\
\text { avenue des Chênes } \\
92360 \text { MEUDON-LA-FORÊT }\end{array}$ \\
\hline ou & $\begin{array}{l}\text { M. SYLVESTRE Jean } \\
\text { rue de la Mésange } \\
88600 \text { BRUYĖRES }\end{array}$ \\
\hline
\end{tabular}

Afin d'éviter la coupure de leur abonnement, nous prions les abonnés dont la mention porte le

$$
n^{\circ} 1
$$

de bien vouloir régler leur cotisation dès la réception de ce numéro, sans attendre la carte de renouvellement habituelle. Le service des abonnements vous remercie d'avance du soulagement qui sera ainsi apporté à la gestion de son fichier. 